\title{
Photocoagulation of subfoveal choroidal neovascular membranes in age related macular degeneration: the impact of the macular photocoagulation study in the United Kingdom and Republic of Ireland
}

Age related macular degeneration (AMD) is the leading cause of blind registration in the developed world. ${ }^{1}$ Choroidal neovascularisation (CNV) leading to haemorrhage and scar formation beneath the central retina accounts for the majority of cases of legal blindness in AMD (80\% to 90\%), the remainder being attributable to atrophic macular changes. ${ }^{2}$

A CNV membrane is described as subfoveal if any part of the lesion lies beneath the centre of the foveal avascular zone (FAZ). The concern regarding laser ablation of subfoveal lesions rests on the fact that photocoagulation of such a CNV membrane necessarily results in destruction of the overlying retina. In 1986, the Macular Photocoagulation Study (MPS) Group initiated a randomised clinical trial to evaluate laser ablation of subfoveal CNV membranes. The inclusion criteria were-a CNV membrane with a classic component and well defined borders; size of lesion $\leq 3.5$ MPS disc areas; and a portion of the CNV membrane directly beneath the geometric centre of the FAZ. ${ }^{3}$ It was found that such patients who were treated according to the MPS protocol had significantly better visual acuity than untreated patients at 48 months' follow up, but that treatment was associated with an immediate average reduction in acuity of 3 Bailey-Lovie lines; the benefits of treatment over observation alone only became apparent 6 months after photocoagulation of the membrane. ${ }^{4}$ However, further analysis of the data showed that laser ablation of small or moderately sized lesions associated with poor acuity ( $\leq 1$ MPS disc area and VA of $6 / 38$ or worse; $>1 \leq 2$ MPS disc areas and VA of $6 / 60$ or worse) had a better visual outcome throughout follow up. ${ }^{4}$

We conducted a survey of all consultant ophthalmologists in the UK and the Republic of Ireland to evaluate the impact of the MPS recommendations on current practice with respect to the management of subfoveal neovascular membranes secondary to AMD. Seven hundred and twenty nine practising consultant ophthalmologists were identified from the registers of the Royal College of Ophthalmologists and the Irish College of Ophthalmologists, and all were invited to complete a confidential questionnaire. Five hundred and eighty $(79.6 \%)$ returned a completed questionnaire, of whom 241 (41.5\%) stated that they do not personally treat CNV membranes as they refer these cases to a colleague with a special interest in macular disease. Of the 339 (58.5\%) ophthalmologists whose practice includes laser photocoagulation of age related CNV membranes, only 46 (13.6\%) stated that they treat subfoveal cases. Reasons given for withholding treatment included: the possibility of an immediate drop in acuity following laser photocoagulation was deemed unacceptable $(215 ; 73.6 \%)$; the evidence of the visual benefits of this treatment was considered unconvincing (173; $59 \%$ ); the risk to benefit ratio of laser therapy was judged to be unfavourable $(157 ; 53.6 \%)$; use of, or referral for, alternative treatment modalities $(17 ; 5.8 \%)$; difficulty in obtaining consent $(10 ; 3.4 \%)$.
The results of this survey demonstrate that practising ophthalmologists in the UK and Ireland remain unconvinced of the benefits of laser ablation of subfoveal CNV membranes secondary to AMD. It is the prerogative of every clinician to refrain from adopting the recommendations of a clinical trial if that evidence base is in conflict with his/her personal experience and clinical judgment. However, our finding that $86.4 \%$ of ophthalmologists who treat age related $\mathrm{CNV}$ membranes choose not to follow the MPS guidelines with respect to subfoveal lesions is surprising for several reasons. Firstly, the randomised clinical trial performed by the MPS Group was exemplary in terms of its scientific design and execution, and was published in an authoritative journal. Secondly, laser ablation of subfoveal CNV membranes in AMD remains the only treatment shown by a randomised clinical trial to improve the visual prognosis of this condition. Thirdly, laser therapy is the only treatment modality to which all ophthalmologists have access. And fourthly, Bernstein and Seddon have shown by means of a hypothetical questionnaire that patients with subfoveal CNV membranes favour the potential long term benefits of laser photocoagulation, in spite of the possible sudden drop in acuity, over the natural course of the disease. ${ }^{5}$

The possibility of precipitating an immediate reduction in acuity was the most frequently cited reason for withholding photocoagulation in cases of subfoveal CNV membranes. As one colleague eloquently put it: "I would rather the patient go blind by the hand of God than by the hand of the ophthalmologist." A firm evidence base of efficacy, although necessary, is insufficient in itself to ensure widespread acceptance of a treatment stratagem. The clinician and patient must both be comfortable with any proposed therapy, and it appears that a fear of actively participating in the inevitable decline in central vision has limited the impact of the MPS recommendations with respect to subfoveal CNV membranes.

It is likely that subspecialty interest influences the ophthalmologist's decision when considering laser ablation of subfoveal CNV membranes, although this is an issue which was not investigated in our survey. Macular and retinal specialists may feel better equipped to offer this treatment because of their familiarity with the current literature in their field and greater experience in the diagnosis and treatment of CNV in association with AMD. However, it is important to recognise that the clinical infrastructure of few, if any, health services would support the referral of all cases of AMD to an ophthalmologist with a special interest in macular disease.

It is clear that alternative treatment modalities for subfoveal CNV membranes need to be developed. Therapies currently under investigation include radiation therapy, photodynamic therapy, drug therapy, macular translocation surgery and submacular surgery with or without transplantation of the retinal pigment epithelium. ${ }^{1}$ 
Laser photocoagulation of subfoveal CNV membranes secondary to AMD is not a panacea, but its superiority over observation alone has been eloquently established by the MPS. Until such time as the benefits of alternative treatment modalities have been demonstrated by randomised controlled trials, patients with subfoveal CNV membranes which meet the MPS eligibility criteria should be made aware of the risks and benefits of laser ablation, and encouraged to make an informed decision about their treatment.

$S$ BEATTY

K G AU EONG

D MCLEOD

P N BISHOP

Academic Department of Ophthalmology, Manchester Royal Eye Hospital, Manchester
Correspondence to: S Beatty, Manchester Royal Eye Hospital, Oxford Road, Manchester M13 9WH

1 Chong NHV, Bird AC. Alternative therapies in exudative age related macular degeneration (Perspective). Br f Ophthalmol 1998;82:1441-3.

2 Leibowitz HM, Krueger DE, Maunder LR, et al. The Framingham Eye Study Monograph. An ophthalmological and epidemiological study of cataract, glaucoma, diabetic retinopathy, macular degeneration and visual acuity in a general population of 2631 adults, 1973-1975. Surv Ophthalmol 1980;24:335-609.

3 Macular Photocoagulation Study Group. Subfoveal neovascular lesions in age related macular degeneration. Guidelines for evaluation and treatment in the macular photocoagulation study. Arch Ophthalmol 1991;109:124257.

4 Macular Photocoagulation Study Group. Visual outcome after laser photocoagulation for subfoveal neovascularization secondary to age-related macular degeneration. The influence of initial lesion size and initial visual acuity. Arch Ophthalmol 1994;112:480-8.

5 Bernstein PS, Seddon JM. Decision-making in the treatment of subfoveal neovascularization in age-related macular degeneration. An analysis from the patient's perspective. Retina 1996;16:112-6. 